\title{
Robust output-feedback controller design via local BMI optimization*
}

\author{
S. Kanev, C. Scherer, M. Verhaegen, and B. De Schutter
}

If you want to cite this report, please use the following reference instead:

S. Kanev, C. Scherer, M. Verhaegen, and B. De Schutter, "Robust output-feedback controller design via local BMI optimization," Automatica, vol. 40, no. 7, pp. 11151127, July 2004.

Delft Center for Systems and Control

Delft University of Technology

Mekelweg 2, 2628 CD Delft

The Netherlands

phone: +31-15-278.24.73 (secretary)

URL: https: //www.dcsc.tudelft.nl

*This report can also be downloaded viahttps://pub.deschutter.info/abs/03_007.html 


\title{
Robust Output-Feedback Controller Design via Local BMI Optimization
}

\author{
S. Kanev ${ }^{a}$, C. Scherer ${ }^{a}$, M. Verhaegen ${ }^{a}$ and B. De Schutter ${ }^{a}$ \\ ${ }^{\text {a }}$ TU-Delft, Delft Center for Systems and Control, \\ Mekelweg 2, 2628 CD Delft, The Netherlands, \\ +31-15-27-86707, fax: +31-15-27-86679
}

\begin{abstract}
The problem of designing a globally optimal full-order output-feedback controller for polytopic uncertain systems is known to be a non-convex NP-hard optimization problem, that can be represented as a bilinear matrix inequality optimization problem for most design objectives. In this paper a new approach is proposed to the design of locally optimal controllers. It is iterative by nature, and starting from any initial feasible controller it performs local optimization over a suitably defined non-convex function at each iteration. The approach features the properties of computational efficiency, guaranteed convergence to a local optimum, and applicability to a very wide range of problems. Furthermore, a fast (but conservative) LMI-based procedure for computing an initially feasible controller is also presented. The complete approach is demonstrated on a model of one joint of a real-life space robotic manipulator.
\end{abstract}

Key words: Multiobjective control, Robust control design, Structured uncertainty, Dynamic output-feedback, LMIs.

\section{Introduction}

In the last decade much research was focused on the development of LMI approaches to controller design (Boyd et al., 1994; Scherer et al., 1997; Gahinet, 1996; Gahinet et al., 1995; Palhares et al., 1996; Oliveira et al., 2002; Kothare et al., 1996), state estimation (Geromel, 1999; Geromel et al., 2000; Geromel and de Oliveira, 2001; Cuzzola and Ferrante, 2001; Palhares et al., 2001), and system performance analysis (Oliveira et al., 1999; Palhares et al., 1997; Zhou et al., 1995) due to the recent development of computationally fast and numerically reliable algorithms for solving convex optimization problems subject to LMI constraints. In the cases when no uncertainty is considered in the model description, numerous LMI-based approaches exist that address the problems of state-feedback (Oliveira et al., 2002; Palhares et al., 1996; Peres and Palhares, 1995) and dynamic output-feedback controller (Apkarian and Gahinet, 1995; Gahinet, 1996; Geromel et al., 1999; Oliveira et al., 2002) design for different design objectives. In these approaches, in general, the controller

Email addresses: S.Kanev@DCSC.TUDelft.NL (S. Kanev), C.W.Scherer@DCSC.TUDelft.NL (C. Scherer),

M.Verhaegen@DCSC.TUDelft.NL (M. Verhaegen),

B.DeSchutter@DCSC. TUDelft.NL (B. De Schutter). state-space matrices are parametrized by a set of matrices representing a feasible solution to a system of LMIs that describes the control objective, plus (often) the state-space matrices of the controlled system. For an overview of the LMI methods for analysis and design of control systems the reader is referred to (Boyd et al., 1994; Scherer et al., 1997) and the references therein.

Whenever the controller parametrization is not explicitly dependent on the state-space matrices of the controlled system, generalization to polytopic uncertainties is trivial. Such cases include the LMI-based statefeedback controller design approaches to $\mathcal{H}_{2}$-control (Palhares et al., 1996; Kothare et al., 1996), $\mathcal{H}_{\infty}$-control (Palhares et al., 1996; Peres and Palhares, 1995; Zhou et al., 1995), pole-placement in LMI regions (Chilali et al., 1999; Scherer et al., 1997), etc. These, however, require that the system state is measurable, thus imposing a severe restriction on the class of systems to which they are applicable.

Similar extension of most of the output-feedback controller design approaches to the structured uncertainty case is, unfortunately, not that simple due to the fact that the controller parametrization explicitly depends on the state-space matrices of the system, which are unknown (Apkarian and Gahinet, 1995; Gahinet, 1996; Ma- 
subuchi et al., 1998; Scherer et al., 1997). Clearly, whenever the uncertainty is unstructured (e.g. high-frequency unmodeled dynamics), it can be recast into the general linear fractional transformation (LFT) representation and using the small gain theorem the design objective can be translated into controller design in the absence of uncertainty (Zhou and Doyle, 1998). Application of this approach to systems with structured uncertainty, i.e. disregarding the structure of the uncertainty, often turns out to be excessively conservative. To overcome this conservatism $\mu$-synthesis was developed (Zhou and Doyle, 1998; Balas et al., 1998), which consists of an iterative procedure (known as $D-K$ iteration) where at each iteration two convex optimizations are executed one in which the controller $K$ is kept fixed, and one in which a certain diagonal scaling matrix $D$ is kept fixed. This procedure, however, is not guaranteed to converge to a local optimum because optimality in two fixed directions does not imply optimality in all possible directions, and it may therefore lead to conservative results (VanAntwerp et al., 1997).

Recently, some attempts have been made towards the development of LMI-based approaches to output-feedback controller design for systems with structured uncertainties in the contexts of robust quadratic stability with disturbance attenuation (Kose and Jabbari, 1999b), linear parameter-varying (LPV) systems (Kose and Jabbari, 1999a), positive real synthesis (Mahmoud and Xie, 2000), and $\mathcal{H}_{\infty}$ control (Xie et al., 1992). In (Kose and Jabbari, 1999b) the authors develop a two-stage procedure for the design of output-feedback controllers for continuous-time systems and provide conditions under which the two stages of the design can be solved sequentially. These conditions, however, restrict the class of systems that can be dealt with by the proposed approach to minimum-phase, left-invertible systems. The same idea has been used in (Kose and Jabbari, 1999a), but extended to deal with LPV systems in which only some of the parameters are measured and the others are treated as uncertainty. In (Mahmoud and Xie, 2000) the output-feedback design of positive real systems is investigated by expressing the uncertainty in an LFT form and recasting the problem to a simplified, but still nonlinear, problem independent of the uncertainties. A possible way, based on eigenvalue assignment, to solve the non-linear optimization problem is proposed that determines the output-feedback controller. This approach is applicable to square systems only. In the case when the uncertainty consists of one full uncertainty block it was shown in (Xie et al., 1992) how the problem can be transformed into a standard $\mathcal{H}_{\infty}$ problem along a line search for a single scalar. However, as argued in (Kose and Jabbari, 1999b), this approach may turn out to be too conservative in cases when the uncertainty contains repeated real scalars.

It is well-known that most of the output-feedback controller design problems are representable in terms of bi- linear (or rather bi-affine) matrix inequalities (BMIs) (VanAntwerp and Braatz, 2000), which however are in general NP-hard (Toker and Özbay, 1995). This means that any algorithm which is guaranteed to find the global optimum cannot be expected to have a polynomial time complexity.

The method proposed in this paper belongs to the class of approaches that directly aim at solving the BMI optimization problem at hand. There exist different approaches to the solution of this problem, which can be classified into global (Beran et al., 1997; Fukuda and Kojima, 2001; Goh et al., 1995; Tuan and Apkarian, 2000; Tuan et al., 2000a,b; VanAntwerp et al., 1997; Yamada and Hara, 1998; Yamada et al., 2001) and local (Ibaraki and Tomizuka, 2001; Iwasaki, 1999; Iwasaki and Rotea, 1997; Hassibi et al., 1999; Grigoradis and Skelton, 1996). Most of the global algorithms to the BMI problem are variations of the Branch and Bound Algorithm (Tuan and Apkarian, 2000; Goh et al., 1995; Fukuda and Kojima, 2001; VanAntwerp et al., 1997; Beran et al., 1997). Although the major focus of all global search algorithms is the computational complexity, none of them is polynomial-time due to the NP-hardness of the problem. As a result, these approaches can currently be applied only to problems of modest size (VanAntwerp et al., 1997) with no more than just a few "complicating variables" ${ }^{1}$ (Tuan and Apkarian, 2000). Thus, the global algorithms are not practical to output-feedback controller design problems for polytopic systems, where even small problems can result in lots of such complicating variables (for instance, in the case study presented in Section $\S 6$ there are 40 complicating variables).

Most of the existing local approaches, on the other hand, are computationally fast but, depending on the initial condition, may not converge to the global optimum. The simplest local approach makes use of the fact that by fixing some of the variables, $\boldsymbol{x}$, the BMI problem becomes convex in the remaining variables $\boldsymbol{y}$, and vice versa, and iterates between them (Iwasaki, 1999). This is also the idea behind the well-known $D-K$ iteration for $\mu$ synthesis (Doyle, 1983). In some papers (Iwasaki, 1999; Iwasaki and Rotea, 1997; Iwasaki and Skelton, 1995) the search is performed in other, more suitably defined search directions. Nevertheless, these type of algorithms, called coordinate descent methods in (Iwasaki, 1999), alternating SDP method in (Fukuda and Kojima, 2001), and the dual iteration in (Iwasaki, 1999), are not guaranteed to converge to a local solution (Goh et al., 1995; Fukuda and Kojima, 2001; Yamada and Hara, 1998).

Recently, interior point methods have also been developed for nonconvex semidefinite programming (SDP)

\footnotetext{
1 Generally speaking, this is the minimal number of variables in the BMI problem that, if kept fixed, results in an LMI problem.
} 
problems (Leibfritz and Mostafa, 2002; Hol et al., 2003; Forsgren, 2000). The interior point approach tries to find an approximate solution to the nonconvex SDP problem by rewriting it as logarithmic barrier function optimization problem. The approach then finds approximate solutions to a sequence of barrier problems and in this way produces an approximate solution to the original nonconvex SDP problem. In (Leibfritz and Mostafa, 2002) a trust region method is proposed for the design of optimal static output-feedback gains. This is a nonconvex BMI problem (Leibfritz, 2001).

Another local approach is the so-called path-following method (Hassibi et al., 1999), which is based on linearization. The idea is that under the assumption of small search steps the BMI problem can be approximated as an LMI problem by making use of the firstorder perturbation approximation (Hassibi et al., 1999). In practice this approach can be used for problems where the required closed-loop performance is not drastically better than the open-loop system performance, to solve the actuator/sensor placement problem, as well as the controller topology design problem (Hassibi et al., 1999). Similar is the continuation algorithm proposed in (Collins et al., 1999) that basically consists in iterating between two LMI problems each obtain by linearization using first order perturbation approximations. Yet another local approach is the rank-minimization method (Ibaraki and Tomizuka, 2001). Although convergence is established for a suitably modified problem, there are no guarantees that the solution to this modified problem will be feasible for the original BMI problem. The $X Y$ centering algorithm, proposed in (Iwasaki and Skelton, 1995 ) is also an alternative local approach, which focusses on a subclass of BMI problems in which the nonconvexity can be expressed in the form $X=Y^{-1}$, and is thus applicable to a restricted class of controller design problems. Finally, the method of centers (MC) (Goh et al., 1995) has guaranteed local convergence provided that a feasible initial condition is given. It is, however, the computationally most involving approach, and it is also known that it can experience numerical problems at later iterations (Fukuda and Kojima, 2001).

Similarly to the MC, the approach in this paper performs local optimization over a suitably defined nonconvex function at each iteration. It enjoys the property of guaranteed convergence to a local optimum, while at the same time is computationally faster and numerically more reliable than the MC. In addition to that, a twostep procedure is proposed for the design of an initially feasible controller. At the first step an optimal robust mixed $\mathcal{H}_{2} / \mathcal{H}_{\infty} /$ pole-placement state-feedback gain $F$ is designed. This gain $F$ is consequently kept fixed during the design of the remaining state-space matrices of the dynamic output-feedback controller. Although the first step is convex, the second one remains non-convex. However, by constraining a Lyapunov function for the closed-loop system to have a block-diagonal structure, this second step is easily transformed into an LMI optimization problem.

The paper is organized as follows. In $\S 2$ the notation is defined and the problem is formulated. The proposed algorithm for locally optimal controller design is next presented in $\S 3$. For the purposes of its initialization, an approach to initially feasible controller computation is proposed in $\S 4$, where a multiobjective criterion is considered. A summary of the complete algorithm is given in $\S 5$. In $\S 6$ the design approach is tested on a case study with a linear model of a space robotic manipulator and, in addition, a comparison is made between several existing methods for local BMI optimization. Finally, $\S 7$ concludes the paper.

\section{Preliminaries and Problem Formulation}

\subsection{Notation}

The symbol • in LMIs will denote entries that follow from symmetry. In addition to that the notation $\operatorname{Sym}(A)=$ $A+A^{*}$ will also be used. Boldface capital letters denote variable matrices appearing in matrix inequalities, and boldface small letters - vector variables. The convex hull of a set of matrices $\mathcal{S}=\left\{M_{1}, \ldots, M_{N}\right\}$ is denoted as $\operatorname{co}\{\mathcal{S}\}$, and is defined as the intersection of all convex sets containing all elements of $\mathcal{S}$. Also used is the notation $\langle A, B\rangle=\operatorname{trace}\left(A^{T} B\right)$ for any matrices $A$ and $B$ of appropriate dimensions, and $\|A\|_{F}$ denotes the Frobenius norm of $A$. The set of eigenvalues of a matrix $A$ will be denoted as $\lambda(A)$, while for a complex number $z \in \mathbb{C}$, the complex conjugate is denoted as $\bar{z}$. The symbol $\triangleq$ will denote "equal by definition". The direct sum of matrices $A_{i}, i=1,2, \ldots, n$ will be denoted as

$$
\bigoplus_{i=1}^{n} A_{i}=A_{1} \oplus \cdots \oplus A_{n} \triangleq \operatorname{diag}\left(A_{1}, A_{2}, \ldots, A_{n}\right)
$$

Also, $v_{i}$ will denote the $i$-th element of the vector $v$. For two matrices $A \in \mathbb{R}^{m \times n}$ and $B \in \mathbb{R}^{p \times q}, A \otimes B \in \mathbb{R}^{m p \times n q}$ denotes the Kronecker product of $A$ and $B$.

The projection onto the cone of symmetric positivedefinite matrices is defined as

$$
[A]^{+}=\arg \min _{\boldsymbol{S} \geq 0}\|A-\boldsymbol{S}\|_{F}
$$

Similarly, the projection onto the cone of symmetric negative-definite matrices is defined as

$$
[A]^{-}=\arg \min _{\boldsymbol{S} \leq 0}\|A-\boldsymbol{S}\|_{F}
$$

This projection has the following properties. 
Lemma 1 (Properties of the projection) For symmetric matrix $A$, the following properties hold

(1) $A=[A]^{+}+[A]^{-}$

(2) $\left\langle[A]^{+},[A]^{-}\right\rangle=0$,

(3) Let $A=U \Lambda U^{T}$, where $U$ is an orthogonal matrix containing the eigenvectors of $A$, and $\Lambda$ is a diagonal matrix with the eigenvalues $\lambda_{i}, \quad i=1, \ldots, n$, of $A$ appearing on its diagonal. Then $[A]^{+}=U \operatorname{diag}\left\{\lambda_{1}^{+}, \ldots, \lambda_{n}^{+}\right\} U^{T}$, with $\lambda_{i}^{+}=\max \left(0, \lambda_{i}\right), i=1, \ldots, n$. Equivalently, $[A]^{-}=U \operatorname{diag}\left\{\lambda_{1}^{-}, \ldots, \lambda_{n}^{-}\right\} U^{T}$, with $\lambda_{i}^{-}=\min \left(0, \lambda_{i}\right), i=1, \ldots, n$.

(4) $[A]^{+}$and $[A]^{-}$are continuous in $A$.

For a proof see (Calafiore and Polyak, 2001).

In the remaining part of this section we summarize some existing results for system analysis and controller synthesis which lie at the basis of the developments in the next Section.

\section{2 $\mathcal{H}_{2}$ and $\mathcal{H}_{\infty}$ Norm Computation for Uncertain Sys-} tems

Consider the uncertain state-space model

$$
\left\{\begin{aligned}
\sigma x & =A^{\Delta} x+B^{\Delta} \xi \\
z & =C^{\Delta} x+D^{\Delta} \xi
\end{aligned}\right.
$$

where $x(t) \in \mathbb{R}^{n}$ is the system state, $z(t) \in \mathbb{R}^{n_{z}}$ is the controlled output of the system, and $\xi(t) \in \mathbb{R}^{n_{\xi}}$ is the disturbance to the system, and where the symbol $\sigma$ represents the $s$-operator (i.e. the time-derivative operator) for continuous-time systems, and the $z$-operator (i.e. the shift operator) for discrete-time systems. Define the matrix

$$
M_{a n}^{\Delta} \triangleq\left[\begin{array}{ll}
A^{\Delta} & B^{\Delta} \\
C^{\Delta} & D^{\Delta}
\end{array}\right]
$$

where the subscript "an" denotes that it will be used for the purposes of analysis only. Later on, a similar matrix for the synthesis problem will be defined. The matrices $\left(A^{\Delta}, B^{\Delta}, C^{\Delta}, D^{\Delta}\right)$ in (3) are assumed unknown, not measurable, but are known to lie in a given convex set $\mathcal{M}_{a n}$, defined as

$$
\mathcal{M}_{a n} \triangleq \mathbf{c o}\left\{\left[\begin{array}{ll}
A_{1} & B_{1} \\
C_{1} & D_{1}
\end{array}\right], \ldots,\left[\begin{array}{ll}
A_{N} & B_{N} \\
C_{N} & D_{N}
\end{array}\right]\right\} .
$$

Next, the transfer function from $\xi$ to $z$ is denoted as

$$
T_{z \xi}^{\Delta}(\sigma) \triangleq C^{\Delta}\left(\sigma I_{n}-A^{\Delta}\right)^{-1} B^{\Delta}+D^{\Delta} .
$$

The following Lemma, which can be found in e.g. (Chilali et al., 1999), can be used to check whether the eigenvalues of a matrix are all located inside an LMI region.

Lemma 2 Let $\mathcal{A}$ be a real matrix, and define the LMI region

$$
\mathcal{D} \triangleq\left\{z \in \mathbb{C}: L_{\mathcal{D}}+\operatorname{Sym}\left(z M_{\mathcal{D}}\right)<0\right\},
$$

for some given real matrices $L_{\mathcal{D}}=L_{\mathcal{D}}^{T}$ and $M_{\mathcal{D}}$. Then $\lambda(\mathcal{A}) \subset \mathcal{D}$ if and only if there exists a matrix $\boldsymbol{P}=\boldsymbol{P}^{T}>0$ such that

$$
L_{\mathcal{D}} \otimes \boldsymbol{P}+\operatorname{Sym}\left(M_{\mathcal{D}} \otimes(\boldsymbol{P} \mathcal{A})\right)<0 .
$$

The class of LMI regions, defined in Equation (8), is fairly general - it can represent convex regions that are symmetric with respect to the real axis (Gahinet et al., 1995).

In (Scherer et al., 1997; Masubuchi et al., 1998) LMI conditions are provided for the evaluation of the $\mathcal{H}_{2}$ and $\mathcal{H}_{\infty}$ norm of the transfer function (6) in the case when there is no uncertainty present in the system, i.e. for the case when the matrix $M_{a n}^{\Delta}$ in (4) is exactly known and time-invariant. The following two results are immediate generalizations to the case when $M_{a n}^{\Delta}$ is only known to lie in a certain convex set $\mathcal{M}_{a n}$, and as such will be left without proof. Define

$$
\begin{aligned}
& \mathcal{L}\left(C^{\Delta}, \boldsymbol{W}, \boldsymbol{P}, \gamma\right)=(\gamma-\operatorname{trace}(\boldsymbol{W})) \oplus\left[\begin{array}{cc}
\boldsymbol{W} & C^{\Delta} \\
\bullet & \boldsymbol{P}
\end{array}\right] \\
& \mathcal{M}_{C T}\left(A^{\Delta}, B^{\Delta}, \boldsymbol{P}\right)=\left[\begin{array}{cc}
-S y m\left(\boldsymbol{P} A^{\Delta}\right) & \boldsymbol{P} B^{\Delta} \\
\bullet & I
\end{array}\right] \\
& \mathcal{M}_{D T}\left(A^{\Delta}, B^{\Delta}, \boldsymbol{P}\right)=\left[\begin{array}{ccc}
\boldsymbol{P} & \boldsymbol{P} A^{\Delta} & \boldsymbol{P} B^{\Delta} \\
\bullet & \boldsymbol{P} & 0 \\
\bullet & \bullet & I
\end{array}\right]
\end{aligned}
$$

Lemma $3\left(\mathcal{H}_{2}\right.$ norm) Assume that $D^{\Delta}=0$. Then

$$
\sup _{M_{a n}^{\Delta} \in \mathcal{M}_{a n}}\left\|T_{z \xi}^{\Delta}(\sigma)\right\|_{2}^{2}<\gamma
$$

if there exist matrices $\boldsymbol{P}=\boldsymbol{P}^{T}$ and $\boldsymbol{W}=\boldsymbol{W}^{T}$ such that for all $M_{a n}^{\Delta} \in \mathcal{M}_{a n}$

$$
\begin{aligned}
& \mathcal{L}\left(C^{\Delta}, \boldsymbol{W}, \boldsymbol{P}, \gamma\right) \oplus \mathcal{M}_{C T}\left(A^{\Delta}, B^{\Delta}, \boldsymbol{P}\right)>0, \\
& \quad(\text { continuous case }), \\
& \mathcal{L}\left(C^{\Delta}, \boldsymbol{W}, \boldsymbol{P}, \gamma\right) \oplus \mathcal{M}_{D T}\left(A^{\Delta}, B^{\Delta}, \boldsymbol{P}\right)>0,
\end{aligned}
$$$$
\text { (discrete case). }
$$ 


\section{Lemma $4\left(\mathcal{H}_{\infty}\right.$ norm $)$}

$$
\sup _{M_{a n}^{\Delta} \in \mathcal{M}_{a n}}\left\|T_{z \xi}^{\Delta}(\sigma)\right\|_{\infty}^{2}<\gamma
$$

if there exists a matrix $\boldsymbol{P}=\boldsymbol{P}^{T}$ such that for all $M_{\text {an }}^{\Delta} \in$ $\mathcal{M}_{\text {an }}$

$\boldsymbol{P} \oplus\left[\begin{array}{c}\mathcal{M}_{C T}\left(A^{\Delta}, B^{\Delta}, \boldsymbol{P}\right)\left[C^{\Delta}, D^{\Delta}\right]^{T} \\ \bullet I\end{array}\right]>0$

(continuous case),

$$
\left[\begin{array}{c}
\mathcal{M}_{D T}\left(A^{\Delta}, B^{\Delta}, \boldsymbol{P}\right)\left[0, C^{\Delta}, D^{\Delta}\right]^{T} \\
\bullet I
\end{array}\right]>0,
$$

(discrete case).

The infinite number of LMIs in Lemmas 3 and 4 over all possible elements of the set $\mathcal{M}_{a n}$ can be substituted by a finite number of LMIs by using the fact that the set $\mathcal{M}_{a n}$ is convex. This can be achieved by substituting the matrices $\left(A_{i}, B_{i}, C_{i}, D_{i}\right)$ from $\left(A^{\Delta}, B^{\Delta}, C^{\Delta}, D^{\Delta}\right)$ in the LMIs (10) and (11), and then searching for a feasible solution for all $i=1, \ldots, N$.

\subsection{Problem Formulation}

We next focus our attention to the synthesis problem. To this end, consider the following uncertain system

$$
\mathcal{S}_{\sigma}:\left\{\begin{aligned}
\sigma x & =A^{\Delta} x+B_{\xi}^{\Delta} \xi+B_{u}^{\Delta} u \\
z & =C_{z}^{\Delta} x+D_{z \xi}^{\Delta} \xi+D_{z u}^{\Delta} u \\
y & =C_{y}^{\Delta} x+D_{y \xi}^{\Delta} \xi+D_{y u}^{\Delta} u
\end{aligned}\right.
$$

where the signals $x, \xi$, and $z$ have the same meaning and the same dimensions as in (3), and where $u \in \mathbb{R}^{m}$ is the control action, and $y \in \mathbb{R}^{p}$ is the measured output.

Similarly as in $\S 2.2$, we define the matrix

$$
M_{s y n}^{\Delta} \triangleq\left[\begin{array}{ccc}
A^{\Delta} & B_{\xi}^{\Delta} & B_{u}^{\Delta} \\
C_{z}^{\Delta} & D_{z \xi}^{\Delta} & D_{z u}^{\Delta} \\
C_{y}^{\Delta} & D_{y \xi}^{\Delta} & D_{y u}^{\Delta}
\end{array}\right]
$$

where the subscript "syn" denotes that it will now be used for the purposes of synthesis. We also define the convex set

$$
\mathcal{M}_{s y n} \triangleq \mathbf{c o}\left\{\left[\begin{array}{ccc}
A_{i} & B_{\xi, i} & B_{u, i} \\
C_{z, i} & D_{z \xi, i} & D_{z u, i} \\
C_{y, i} & D_{y \xi, i} & D_{y u, i}
\end{array}\right]: i=1, \ldots, N\right\}
$$

Interconnected to system (12) is the following full-order dynamic output-feedback controller

$$
\mathcal{C}_{\sigma}:\left\{\begin{aligned}
\sigma x^{c} & =A_{c} x^{c}+B_{c} y \\
u & =F x^{c}
\end{aligned}\right.
$$

with $x^{c} \in \mathbb{R}^{n}$ its state. This yields the closed-loop system

$$
\left\{\begin{aligned}
\sigma \tilde{x} & =A_{c l}^{\Delta} \tilde{x}+B_{c l}^{\Delta} \xi \\
z & =C_{c l}^{\Delta} \tilde{x}+D_{c l}^{\Delta} \xi
\end{aligned}\right.
$$

where it is denoted $\tilde{x}^{T}=\left[x^{T},\left(x^{c}\right)^{T}\right]$, and

$$
\left[\begin{array}{c|c}
A_{c l}^{\Delta} & B_{c l}^{\Delta} \\
\hline C_{c l}^{\Delta} & D_{c l}^{\Delta}
\end{array}\right] \triangleq\left[\begin{array}{cc|c}
A^{\Delta} & B_{u}^{\Delta} F & B_{\xi}^{\Delta} \\
B_{c} C_{y}^{\Delta} & A_{c}+B_{c} D_{y u}^{\Delta} F & B_{c} D_{y \xi}^{\Delta} \\
\hline C_{z}^{\Delta} & D_{z u}^{\Delta} F & D_{z \xi}^{\Delta}
\end{array}\right] .
$$

Denoting the transfer function from the disturbance $\xi$ to the controlled output $z$, corresponding to the statespace model (16), as

$$
T_{c l}^{\Delta}(\sigma) \triangleq C_{c l}^{\Delta}\left(\sigma I_{2 n}-A_{c l}^{\Delta}\right)^{-1} B_{c l}^{\Delta}+D_{c l}^{\Delta},
$$

this paper addresses the following problem:

Multiobjective Design: Given positive scalars $\alpha_{2}$ and $\alpha_{\infty}$ and a convex set $\mathcal{M}$, defined in Equation (7), find constant matrices $A_{c}, B_{c}$, and $F$, parametrizing the controller (15), that solve the following constrained optimization problem

$$
\begin{array}{ll} 
& \min _{\gamma_{2}, \gamma_{\infty}, A_{c}, B_{c}, F} \alpha_{2} \gamma_{2}+\alpha_{\infty} \gamma_{\infty} \\
\text { s.t. } & \\
\mathcal{H}_{2}: & \sup _{M_{s y n}^{\Delta} \in \mathcal{M}_{s y n}}\left\|L_{2}\left(T_{c l}^{\Delta}(\sigma)-D_{c l}^{\Delta}\right) R_{2}\right\|_{2}^{2}<\gamma_{2} \\
\mathcal{H}_{\infty}: & \sup _{M_{s y n}^{\Delta} \in \mathcal{M}_{s y n}}\left\|L_{\infty} T_{c l}^{\Delta}(\sigma) R_{\infty}\right\|_{\infty}^{2}<\gamma_{\infty} \\
\text { PP: } & \lambda\left(A_{c l}^{\Delta}\right) \in \mathcal{D}, \forall M_{s y n}^{\Delta} \in \mathcal{M}_{s y n}
\end{array}
$$

where the matrices $L_{2}, R_{2}, L_{\infty}$, and $R_{\infty}$, are used to select the desired input-output channels that need to satisfy the required constraint in (19).

As discussed in the introduction, this problem is not convex and is NP-hard. In the next section we will present a new algorithm which can be used for finding a locally optimal solution to the problem defined in (19). As most local approaches, this approach requires an initially feasible solution from which the local optimization is initiated. For the purposes of its initialization, a computationally fast approach based on LMIs for finding an initially feasible controller is later on proposed in $\S 4$. A summary of the complete algorithm is given in $\S 5$. 


\section{Locally Optimal Robust Controller Design}

It is well-known that for systems with polytopic uncertainty the output-feedback controller design problem can be written as BMIs in the general form (21) (VanAntwerp and Braatz, 2000). In this section a method for solving BMI problems is proposed. To this end, define the following $N$ biaffine functions

$$
\begin{aligned}
& \mathcal{B M}^{(k)}(\boldsymbol{x}, \boldsymbol{y}) \triangleq F_{00}^{(k)}+ \\
& \sum_{i=1}^{N_{1}} F_{i 0}^{(k)} \boldsymbol{x}_{i}+\sum_{j=1}^{N_{2}} F_{0 j}^{(k)} \boldsymbol{y}_{j}+\sum_{i=1}^{N_{1}} \sum_{j=1}^{N_{2}} F_{i j}^{(k)} \boldsymbol{x}_{i} \boldsymbol{y}_{j},
\end{aligned}
$$

where $F_{i j}^{(k)}=\left(F_{i j}^{(k)}\right)^{T}, i=0,1, \ldots, N_{1}, j=0,1, \ldots, N_{2}$, $k=1, \ldots, M$ are given symmetric matrices. In this paper we consider the following BMI optimization problem

$$
(\mathcal{P}):\left\{\begin{array}{c}
\min \gamma, \text { over } \boldsymbol{x}, \boldsymbol{y}, \text { and } \gamma \\
\text { s.t. } \mathcal{B M}^{(k)}(\boldsymbol{x}, \boldsymbol{y}) \leq 0, \quad k=1, \ldots, M, \\
\langle c, \boldsymbol{x}\rangle+\langle d, \boldsymbol{y}\rangle \leq \gamma, \\
\underline{x} \leq \boldsymbol{x} \leq \bar{x}, \underline{y} \leq \boldsymbol{y} \leq \bar{y}
\end{array}\right.
$$

where $\underline{x}, \bar{x} \in \mathbb{R}^{N_{1}}$ and $y, \bar{y} \in \mathbb{R}^{N_{2}}$ are given vectors with finite elements. This problem is known to be NP-hard (Toker and Özbay, 1995). The bounds on the variables $\boldsymbol{x}$ and $\boldsymbol{y}$ in (21) are included here for technical reasons that will become clear shortly. The problem of selecting these bounds in practice is not critical - taking the upper bounds large enough (e.g. $10^{10}$ ), and the lower bounds small enough is often sufficient. Notice that in this way one could also ensure, for implementation reasons, that the resulting controller does not have excessively large entries in its state-space matrices.

It should also be pointed out that the BMI problem defined in (21) actually addresses a wider class of problems than those represented by (19), e.g. the design of reduced order output-feedback control (Goh et al., 1996). However, the focus of the paper is restricted to (19) since the initial controller design method, discussed later on in $\S 4$, is developed only for the case of full order outputfeedback control problems.

Let us, for now, consider the feasibility problem for a fixed $\gamma$. Denote

$$
\begin{aligned}
& \mathcal{B M I}^{(M+1)}(\boldsymbol{x}, \boldsymbol{y}) \triangleq\langle c, \boldsymbol{x}\rangle+\langle d, \boldsymbol{y}\rangle-\gamma, \\
& \mathcal{B} \mathcal{M} \mathcal{I}^{(M+2)}(\boldsymbol{x}, \boldsymbol{y}) \triangleq \boldsymbol{x}-\bar{x}, \mathcal{B M}^{(M+3)}(\boldsymbol{x}, \boldsymbol{y}) \triangleq x-\boldsymbol{x}, \\
& \mathcal{B} \mathcal{M} \mathcal{I}^{(M+4)}(\boldsymbol{x}, \boldsymbol{y}) \triangleq \boldsymbol{y}-\bar{y}, \mathcal{B M}^{(M+5)}(\boldsymbol{x}, \boldsymbol{y}) \triangleq \underline{y}-\boldsymbol{y}
\end{aligned}
$$

and let $N=M+5$. The feasibility problem is then defined as

$$
(\mathcal{F P}):\left\{\begin{array}{l}
\text { Find }(\boldsymbol{x}, \boldsymbol{y}) \\
\text { such that } \bigoplus_{k=1}^{N} \mathcal{B M}^{(k)}(\boldsymbol{x}, \boldsymbol{y}) \leq 0
\end{array}\right.
$$

Define the following cost function

$$
v_{\gamma}(\boldsymbol{x}, \boldsymbol{y}) \triangleq\left\|\left[\bigoplus_{k=1}^{N} \mathcal{B M}^{(k)}(\boldsymbol{x}, \boldsymbol{y})\right]^{+}\right\|_{F}^{2} \geq 0
$$

From the definition of the projection [.] ${ }^{+}$, and from the properties of the Frobenius norm we can write

$$
\begin{aligned}
& v_{\gamma}(\boldsymbol{x}, \boldsymbol{y})= \\
& \sum_{k=1}^{N}\left\|\left[\mathcal{B M}^{(k)}(\boldsymbol{x}, \boldsymbol{y})\right]^{+}\right\|_{F}^{2} \triangleq \sum_{k=1}^{N} v_{\gamma}^{(k)}(\boldsymbol{x}, \boldsymbol{y})
\end{aligned}
$$

It is therefore clear that

$$
(\mathcal{F P}) \text { is feasible } \Leftrightarrow 0=\min _{\boldsymbol{x}, \boldsymbol{y}} v_{\gamma}(\boldsymbol{x}, \boldsymbol{y}) .
$$

In this way we have rewritten the BMI feasibility problem $(\mathcal{F P})$ as an optimization problem, where the goal is now to find a local minimum of $v_{\gamma}$. However, the function $v_{\gamma}(\boldsymbol{x}, \boldsymbol{y})$ is not convex. Even worse, it may have multiple local minima. Now, if $\left(\boldsymbol{x}_{\text {opt }}, \boldsymbol{y}_{\text {opt }}\right)$ is a local minimum for $v_{\gamma}$ and is such that $v_{\gamma}\left(\boldsymbol{x}_{\text {opt }}, \boldsymbol{y}_{\text {opt }}\right)=0$, then $\left(\boldsymbol{x}_{\text {opt }}, \boldsymbol{y}_{\text {opt }}\right)$ is also a feasible solution to $(\mathcal{F} \mathcal{P})$. However, if $\left(\boldsymbol{x}_{\text {opt }}, \boldsymbol{y}_{\text {opt }}\right)$ is such that $v_{\gamma}\left(\boldsymbol{x}_{\text {opt }}, \boldsymbol{y}_{\text {opt }}\right)>0$, then we cannot say anything about the feasibility of $(\mathcal{F P})$. The idea is then to start from a feasible solution for a given $\gamma$, and then apply the method of bisection over $\gamma$ to achieve a local minimum with a desired precision, at each iteration searching for a feasible solution to $(\mathcal{F P})$. A more extensive description of this bisection algorithm is provided in $\S 5$.

Let us now concentrate on the problem of finding a local solution to

$$
\min _{\boldsymbol{x}, \boldsymbol{y}} v_{\gamma}(\boldsymbol{x}, \boldsymbol{y})
$$

The goal is to develop an approach that has a guaranteed convergence to a local optimum of $v_{\gamma}(\boldsymbol{x}, \boldsymbol{y})$. To this end, we first note that the function $v_{\gamma}(\boldsymbol{x}, \boldsymbol{y})$ is differentiable, and we derive an expression for its gradient.

Theorem 5 With continuously differentiable $G$ : $\mathbb{R}^{N_{v}} \mapsto \mathbb{R}^{q \times q}, G=G^{T}$, and $f: \mathbb{R}^{q \times q} \mapsto \mathbb{R}$ defined as $f(M)=\left\|[M]^{+}\right\|_{F}^{2}$, the function

$$
(f \circ G)(\boldsymbol{v}) \triangleq\left\|[G(\boldsymbol{v})]^{+}\right\|_{F}^{2},
$$


is differentiable, and its gradient

$$
\nabla(f \circ G)(\boldsymbol{v}) \triangleq\left[\frac{\partial}{\partial \boldsymbol{v}_{1}}, \frac{\partial}{\partial \boldsymbol{v}_{2}}, \ldots \frac{\partial}{\partial \boldsymbol{v}_{N_{v}}}\right]^{T}(f \circ G)(\boldsymbol{v}),
$$

is given by

$$
\frac{\partial}{\partial \boldsymbol{v}_{i}}(f \circ G)(\boldsymbol{v})=2\left\langle[G(\boldsymbol{v})]^{+}, \frac{\partial}{\partial \boldsymbol{v}_{i}} G(\boldsymbol{v})\right\rangle .
$$

PROOF. Using the properties of the projection [.] $]^{+}$we infer for any symmetric matrices $G$ and $\Delta G$, that

$$
\begin{aligned}
& f \circ(G+\Delta G)=\left\|G+\Delta G-[G+\Delta G]^{-}\right\|_{F}^{2} \\
& =\min _{S \leq 0}\|G+\Delta G-S\|_{F}^{2} \leq\left\|G+\Delta G-[G]^{-}\right\|_{F}^{2} \\
& =\left\|[G]^{+}+\Delta G\right\|_{F}^{2}=\left\|[G]^{+}\right\|_{F}^{2}+2\left\langle[G]^{+}, \Delta G\right\rangle+\|\Delta G\|_{F}^{2} .
\end{aligned}
$$

On the other hand,

$$
\begin{aligned}
& f \circ(G+\Delta G)=\left\|G+\Delta G-[G+\Delta G]^{-}\right\|_{F}^{2} \\
& =\left\|[G]^{+}+[G]^{-}+\Delta G-[G+\Delta G]^{-}\right\|_{F}^{2} \\
& \geq\left\|[G]^{+}\right\|_{F}^{2}+2\left\langle[G]^{+}, \Delta G\right\rangle+2\left\langle[G]^{+},[G]^{-}\right\rangle \\
& +2\left\langle[G]^{+},-[G+\Delta G]^{-}\right\rangle \geq\left\|[G]^{+}\right\|_{F}^{2}+2\left\langle[G]^{+}, \Delta G\right\rangle .
\end{aligned}
$$

Thus we have $f \circ(G+\Delta G)=f \circ G+2\left\langle[G]^{+}, \Delta G\right\rangle+$ $o\left(\|\Delta G\|_{F}\right)$ for any symmetric $\Delta G$.

Now, take $\Delta G(\boldsymbol{v}) \triangleq G(\boldsymbol{v}+\Delta \boldsymbol{v})-G(\boldsymbol{v})$. Since $G(\boldsymbol{v})$ is continuously differentiable it follows that

$G(\boldsymbol{v}+\Delta \boldsymbol{v})=G(\boldsymbol{v})+\sum_{i=1}^{N_{v}}\left(\frac{\partial}{\partial \boldsymbol{v}_{i}} G(\boldsymbol{v})\right) \Delta \boldsymbol{v}_{i}+o\left(\|\Delta \boldsymbol{v}\|_{2}\right)$

Therefore

$$
\begin{aligned}
& (f \circ G)(\boldsymbol{v}+\Delta \boldsymbol{v})=(f \circ G)(\boldsymbol{v})+ \\
& 2 \sum_{i=1}^{N_{v}}\left(\left\langle[G(\boldsymbol{v})]^{+}, \frac{\partial}{\partial \boldsymbol{v}_{i}} G(\boldsymbol{v})\right\rangle \Delta \boldsymbol{v}_{\boldsymbol{i}}\right)+o\left(\|\Delta \boldsymbol{v}\|_{2}\right) .
\end{aligned}
$$

Hence $(f \circ G)$ is differentiable and its partial derivatives are given by the expressions (26).

The partial derivatives of the function $v_{\gamma}(\boldsymbol{x}, \boldsymbol{y})$ can then be directly derived using the result of Theorem 5 :

$$
\begin{aligned}
& \frac{\partial}{\partial \boldsymbol{x}_{i}} v_{\gamma}(\boldsymbol{x}, \boldsymbol{y})=2 \sum_{k=1}^{N}\left\langle\left[\mathcal{B} \mathcal{M} \mathcal{I}^{(k)}(\boldsymbol{x}, \boldsymbol{y})\right]^{+}, F_{i 0}^{(k)}+\sum_{j=1}^{N_{2}} F_{i j}^{(k)} \boldsymbol{y}_{j}\right\rangle \\
& \frac{\partial}{\partial \boldsymbol{y}_{j}} v_{\gamma}(\boldsymbol{x}, \boldsymbol{y})=2 \sum_{k=1}^{N}\left\langle\left[\mathcal{B} \mathcal{M} \mathcal{I}^{(k)}(\boldsymbol{x}, \boldsymbol{y})\right]^{+}, F_{0 j}^{(k)}+\sum_{i=1}^{N_{1}} F_{i j}^{(k)} \boldsymbol{x}_{i}\right\rangle
\end{aligned}
$$

Note that these partial derivatives are continuous functions (see Lemma 1 ), so that $v_{\gamma} \in C^{1}$. Note also, that a lower bound on the cost function in (21) can always be obtained by solving the so-called relaxed LMI optimization problem (Tuan and Apkarian, 2000)

$$
\gamma_{L B}=\min _{\boldsymbol{x}, \boldsymbol{y}}\langle c, \boldsymbol{x}\rangle+\langle d, \boldsymbol{y}\rangle
$$$$
\text { subject to: } \boldsymbol{x} \in[\underline{x}, \bar{x}], \boldsymbol{y} \in[\underline{y}, \bar{y}], \boldsymbol{w}_{i j} \in\left[\underline{w}_{i j}, \bar{w}_{i j}\right]
$$

$$
F_{00}^{(k)}+\sum_{i=1}^{N_{1}} F_{i 0}^{(k)} \boldsymbol{x}_{i}+\sum_{j=1}^{N_{2}} F_{0 j}^{(k)} \boldsymbol{y}_{j}+
$$

$$
\sum_{i=1}^{N_{1}} \sum_{j=1}^{N_{2}} F_{i j}^{(k)} \boldsymbol{w}_{i j} \leq 0 \text { for } k=1,2, \ldots, M
$$

where $\underline{w}_{i j}=\min \left\{\underline{x}_{i} \underline{y}_{j}, \underline{x}_{i} \bar{y}_{j}, \bar{x}_{i} \underline{y}_{j}, \bar{x}_{i} \bar{y}_{j}\right\}$, and $\bar{w}_{i j}=$ $\max \left\{\underline{x}_{i} \underline{y}_{j}, \underline{x}_{i} \bar{y}_{j}, \bar{x}_{i} \underline{y}_{j}, \bar{x}_{i} \bar{y}_{j}\right\}$.

If this problem is not feasible, then the original BMI problem is also not feasible.

Now that it was shown that the function $v_{\gamma}$ is $C^{1}$ and an expression for its gradient has been derived, the cautious BFGS method (Li and Fukushima, 2001), a quasiNewton type optimization algorithm, could be used for finding a local minimum of $v_{\gamma} \in C^{1}$. The convergence of this algorithm is established in ( $\mathrm{Li}$ and Fukushima, 2001) under the assumption that, (a) the level set $\Omega=$ $\left.\left\{\boldsymbol{x}, \boldsymbol{y}: \quad v_{\gamma}(\boldsymbol{x}, \boldsymbol{y}) \leq v_{\gamma}\left(\boldsymbol{x}^{(\mathbf{0})}, \boldsymbol{y}^{(\mathbf{0})}\right)\right)\right\}$ is bounded, (b) $v_{\gamma}(\boldsymbol{x}, \boldsymbol{y})$ is continuously differentiable on $\Omega$, and (c) there exists a constant $L>0$ such that the global Lipschitz condition holds:

$$
\|g(\boldsymbol{x}, \boldsymbol{y})-g(\overline{\boldsymbol{x}}, \overline{\boldsymbol{y}})\|_{2} \leq L\left\|\left[\begin{array}{l}
\boldsymbol{x}-\overline{\boldsymbol{x}} \\
\boldsymbol{y}-\overline{\boldsymbol{y}}
\end{array}\right]\right\|_{2}, \forall(\boldsymbol{x}, \boldsymbol{y}),(\overline{\boldsymbol{x}}, \overline{\boldsymbol{y}}) \in \Omega .
$$

For the problem considered in this section the level set $\Omega$ is compact (see equation (21)), so that condition (a) holds. Condition (b) was shown in Theorem 5. Condition (c) follows by observing that the projection [.. $]^{+}$is Lipschitz, and hence, since $\mathcal{B M I}^{(k)}(\boldsymbol{x}, \boldsymbol{y})$ is smooth, the functions in partial derivatives $\partial v_{\gamma} / \partial \boldsymbol{x}_{i}$ and $\partial v_{\gamma} / \partial \boldsymbol{y}_{j}$ satisfy a local Lipschitz condition. The compactness of the set $\Omega$ then implies the desired global Lipschitz condition.

Note that the optimization problem discussed above applies to a more general class of problems with smooth nonlinear matrix inequality (NMI) constraints. However, finding an initially feasible solution to start the local optimization is a rather difficult problem, for which reason NMI problems fall outside the scope of this paper. It also needs to be noted here that any algorithm with guaranteed convergence to a local minimum could be used instead of the BFGS algorithm. 
In the next section we focus on the problem of finding an initial feasible solution to the BMI optimization problem.

\section{Initial Robust Multiobjective Controller De- sign}

In this Section, a two-step procedure is presented for the design of an initial feasible robust output-feedback controller. It can be summarized as follows:

Step 1: Design a robust state-feedback gain matrix $\boldsymbol{F}$ such that the multiobjective criterion of the form (19) is satisfied for the closed-loop system with statefeedback control $u=F x$. This problem is convex and is considered in Subsection $\S 4.1$.

Step 2: Plug the state-feedback gain matrix $F$, computed at Step 1, into the original closed-loop system (16), and search for a solution to the multiobjective control problem, defined in Equation (19), in terms of the remaining unknown controller matrices $\boldsymbol{A}_{\boldsymbol{c}}$ and $\boldsymbol{B}_{\boldsymbol{c}}$. This problem, in contrast to the one in Step 1 above, remains non-convex. It is discussed in $\S 4.2$.

In the remaining part of this Section we proceed with proposing a solution to the problems in the two steps above.

\subsection{Step 1: Robust Multiobjective State-Feedback De- sign}

The state-feedback case for the system (12) is equivalent to taking $C_{y}^{\Delta}=I_{n}, D_{y \xi}^{\Delta}=0_{n \times n_{\xi}}, D_{y u}^{\Delta}=0_{n \times m}$, so that $y \equiv x$. Furthermore, we consider the constant statefeedback controller $u=F x$, which results in the closedloop transfer function

$$
\begin{aligned}
& \tilde{T}_{c l}^{\Delta}(\sigma) \triangleq D_{z \xi}^{\Delta}+ \\
& \left(C_{z}^{\Delta}+D_{z u}^{\Delta} F\right)\left(\sigma I_{n}-\left(A^{\Delta}+B_{u}^{\Delta} F\right)\right)^{-1} B_{\xi}^{\Delta} .
\end{aligned}
$$

The following Theorem can be used for robust multiobjective state-feedback design for discrete-time and continuous-time systems. The proof follows after rewriting Lemmas 3 and 4 for the closed-loop system (28) as LMIs in $\boldsymbol{Q}=\boldsymbol{P}^{-1}$, with subsequent change of variables. It will be omitted here (Scherer et al., 1997; Oliveira et al., 2002).

Theorem 6 (State-Feedback Case) Consider the system (12), and assume that $C_{y}^{\Delta}=I_{n}, D_{y \xi}^{\Delta}=0_{n \times n_{\xi}}$, $D_{y u}^{\Delta}=0_{n \times m}$. Consider the controller $u=F x$ resulting in the closed-loop transfer function $\tilde{T}_{c l}^{\Delta}(\sigma)$, defined in
(28). Given $L_{2}, R_{2}, L_{\infty}$, and $R_{\infty}$, the conditions

$$
\begin{aligned}
& \sup _{M_{s y n}^{\Delta} \in \mathcal{M}_{s y n}}\left\|L_{2}\left(\tilde{T}_{c l}^{\Delta}(\sigma)-D_{z \xi}^{\Delta}\right) R_{2}\right\|_{2}^{2}<\gamma_{2}, \\
& \sup _{M_{s y n}^{\Delta} \in \mathcal{M}_{s y n}}\left\|L_{\infty} \tilde{T}_{c l}^{\Delta}(\sigma) R_{\infty}\right\|_{\infty}^{2}<\gamma_{\infty}, \\
& \lambda\left(A^{\Delta}+B_{u}^{\Delta} F\right) \in \mathcal{D}, \forall M_{s y n}^{\Delta} \in \mathcal{M}_{s y n} .
\end{aligned}
$$

hold if there exist matrices $\boldsymbol{Q}=\boldsymbol{Q}^{T}, \boldsymbol{W}=\boldsymbol{W}^{T}, \boldsymbol{R}=$ $\boldsymbol{R}^{T}$, and $\boldsymbol{L}$ such that for all $i=1, \ldots, N$ the following LMIs hold

$$
\begin{aligned}
& P P:(-\boldsymbol{Q}) \oplus\left(L_{\mathcal{D}} \otimes \boldsymbol{Q}+\operatorname{Sym}\left(M_{\mathcal{D}} \otimes \mathbb{A}_{i}\right)\right)<0 \\
& \mathcal{H}_{2}:\left(\gamma_{2}-\operatorname{trace}(\boldsymbol{R})\right) \oplus\left[\begin{array}{cc}
\boldsymbol{R} & L_{2} \mathbb{C}_{i} \\
\bullet & \boldsymbol{Q}
\end{array}\right] \oplus \\
& \left\{\begin{array}{l}
{\left[\begin{array}{cc}
-\operatorname{Sym}\left(\mathbb{A}_{i}\right) & B_{\xi, i} R_{2} \\
\bullet & I
\end{array}\right]>0, \quad \text { (cont. case) }} \\
{\left[\begin{array}{ccc}
\boldsymbol{Q} & \mathbb{A}_{i} & B_{\xi, i} R_{2} \\
\bullet & \boldsymbol{Q} & 0 \\
\bullet & \bullet & I
\end{array}\right]>0, \quad \text { (discr. case). }}
\end{array}\right.
\end{aligned}
$$

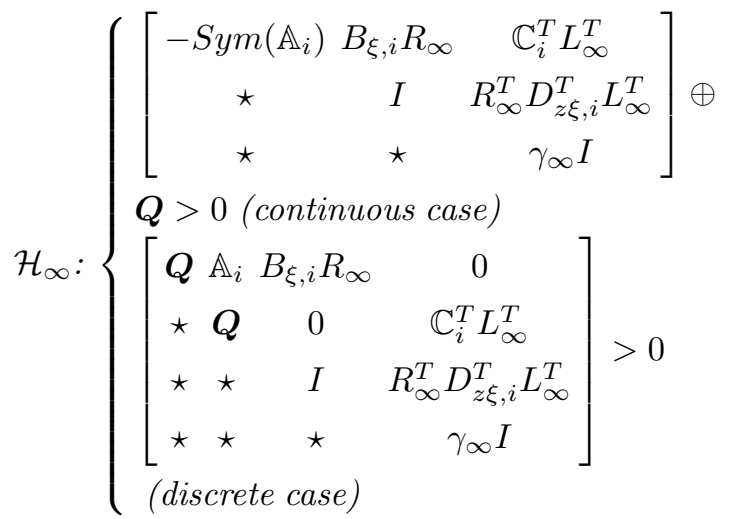

where $\mathbb{A}_{i} \triangleq A_{i} \boldsymbol{Q}+B_{u, i} \boldsymbol{L}$ and $\mathbb{C}_{i} \triangleq C_{z, i} \boldsymbol{Q}+D_{z u, i} \boldsymbol{L}$. The state-feedback gain matrix $F$ is then given by $F=\boldsymbol{L} \boldsymbol{Q}^{-1}$.

\subsection{Step 2: Robust Multiobjective Output-Feedback De- sign}

In what follows we assume that the optimal statefeedback gain $F$ has already been computed at Step 1. In contrast to Step 1, the problem defined in Step 2 of the algorithm at the beginning of $\S 4$ is certainly non-convex in the variables $\boldsymbol{P}, \boldsymbol{W}, \boldsymbol{A}_{\boldsymbol{c}}$, and $\boldsymbol{B}_{\boldsymbol{c}}$ since application of Lemmas 3 and 4 to the closed-loop system in Equation (17) leads to non-linear matrix inequalities due to the fact that the variables $\boldsymbol{A}_{\boldsymbol{c}}$ and $\boldsymbol{B}_{\boldsymbol{c}}$ appear in the closed-loop system matrices $\boldsymbol{A}_{c l}^{\Delta}$ and $\boldsymbol{B}_{c l}^{\Delta}$ (for which reason the last two are typed in boldface). 
Note that the function $V=\tilde{x}^{T} \boldsymbol{P} \tilde{x}$ acts as a Lyapunov function for the closed-loop system. This can easily be seen by observing that the matrix inequalities in Lemmas 3 and 4, when applied to the closed-loop system (16) imply $\left(\boldsymbol{A}_{c l}^{\Delta}\right)^{T} \boldsymbol{P} \boldsymbol{A}_{c l}^{\Delta}-\boldsymbol{P}<0$ for the discrete-time case, and $\boldsymbol{P} \boldsymbol{A}_{c l}^{\Delta}+\left(\boldsymbol{A}_{c l}^{\Delta}\right)^{T} \boldsymbol{P}<0$ for the continuous-time case.

The purpose of this section is to show how by introducing some conservatism by means of constraining the Lyapunov matrix $\boldsymbol{P}$ to have block-diagonal structure

$$
\boldsymbol{P}=\boldsymbol{X} \oplus \boldsymbol{Y}
$$

the nonlinear matrix inequalities in question can be written as LMIs. However, it can easily be seen that a necessary condition for the existence of a structured Lyapunov matrix of the form (33) for $\boldsymbol{A}_{c l}^{\Delta}$ defined in (17) is that the matrix $A^{\Delta}$ is stable for all $M_{s y n}^{\Delta} \in \mathcal{M}_{s y n}$. Luckily, this restriction can be removed by introducing a change of basis of the state vector of the closed-loop system

$$
\bar{x}=T \tilde{x}=\left[\begin{array}{c}
x \\
x-x^{c}
\end{array}\right] .
$$

This changes the state-space matrices of the closed-loop system to

$$
\begin{aligned}
& \overline{\boldsymbol{A}}_{c l}^{\Delta}=T \boldsymbol{A}_{c l}^{\Delta} T= \\
& {\left[\begin{array}{cc}
A^{\Delta}+B_{u}^{\Delta} F & -B_{u}^{\Delta} F \\
A^{\Delta}+B_{u}^{\Delta} F-B_{c} C_{y}^{\Delta}-A_{c}-B_{c} D_{y u}^{\Delta} F & \boldsymbol{A}_{c}+B_{c} D_{y u}^{\Delta} F-B_{u}^{\Delta} F
\end{array}\right]} \\
& \overline{\boldsymbol{B}}_{c l}^{\Delta}=T \boldsymbol{B}_{c l}^{\Delta}=\left[\begin{array}{c}
B_{\xi}^{\Delta} \\
B_{\xi}^{\Delta}-B_{c} D_{y \xi}^{\Delta}
\end{array}\right] \\
& \bar{C}_{c l}^{\Delta}=C_{c l}^{\Delta} T=\left[\begin{array}{c}
C_{z}^{\Delta}+D_{z u}^{\Delta} F-D_{z u}^{\Delta} F
\end{array}\right] \\
& \bar{D}_{c l}^{\Delta}=D_{c l}^{\Delta}=D_{z \xi}^{\Delta}
\end{aligned}
$$

Now, searching for a structured Lyapunov matrix for this (equivalent) closed-loop system only necessitates the stability of the matrix $\left(A^{\Delta}+B_{u}^{\Delta} F\right)$ for all $M_{s y n}^{\Delta} \in$ $\mathcal{M}_{\text {syn }}$, which is guaranteed to hold by the design of the state-feedback gain $F$.

We are now ready to present the following result.

Theorem 7 (Output-Feedback Case) Consider the closed-loop system (16), with transfer function $T_{c l}^{\Delta}(\sigma)$ defined in Equation (18), formed by interconnecting the plant (12) with the dynamic output-feedback controller (15), in which the state-feedback gain matrix $F$ is given. Then given matrices $L_{2}, R_{2}, L_{\infty}$, and $R_{\infty}$ of appropriate dimensions, the conditions

$$
\begin{aligned}
& \sup _{M_{a n}^{\Delta} \in \mathcal{M}_{a n}}\left\|L_{2}\left(T_{c l}^{\Delta}(\sigma)-D_{c l}^{\Delta}\right) R_{2}\right\|_{2}^{2}<\gamma_{2}, \\
& \sup _{M_{a n}^{\Delta} \in \mathcal{M}_{a n}}\left\|L_{\infty} T_{c l}^{\Delta}(\sigma) R_{\infty}\right\|_{\infty}^{2}<\gamma_{\infty}, \\
& \lambda\left(A_{c l}^{\Delta}\right) \in \mathcal{D}, \forall M_{a n}^{\Delta} \in \mathcal{M}_{a n} .
\end{aligned}
$$

hold if there exist matrices $\boldsymbol{W}=\boldsymbol{W}^{T}, \boldsymbol{X}=\boldsymbol{X}^{T}, \boldsymbol{Y}=$ $\boldsymbol{Y}^{T}, \boldsymbol{Z}$ and $\boldsymbol{G}$ such that the following system of LMIs has a feasible solution for all $i=1, \ldots, N$

$$
\begin{aligned}
& P P:(-\boldsymbol{P}) \oplus\left(L_{\mathcal{D}} \otimes \boldsymbol{P}+\operatorname{Sym}\left(M_{\mathcal{D}} \otimes \boldsymbol{M}_{i}\right)\right)<0, \\
& \mathcal{H}_{2}:\left(\gamma_{2}-\operatorname{trace}(\boldsymbol{W})\right) \oplus\left[\begin{array}{cc}
\boldsymbol{W} & L_{2} \Omega_{i} \\
\bullet & \boldsymbol{P}
\end{array}\right] \oplus \\
& \left\{\begin{array}{l}
{\left[\begin{array}{cc}
-\operatorname{Sym}\left(\boldsymbol{M}_{i}\right) & \boldsymbol{N}_{i} R_{2} \\
\bullet & I
\end{array}\right]>0 \quad \text { (cont. case) }} \\
{\left[\begin{array}{ccc}
\boldsymbol{P} & \boldsymbol{M}_{i} & \boldsymbol{N}_{i} R_{2} \\
\bullet & \boldsymbol{P} & 0 \\
\bullet & \bullet & I
\end{array}\right]>0} \\
\text { (discr. case). }
\end{array}\right.
\end{aligned}
$$

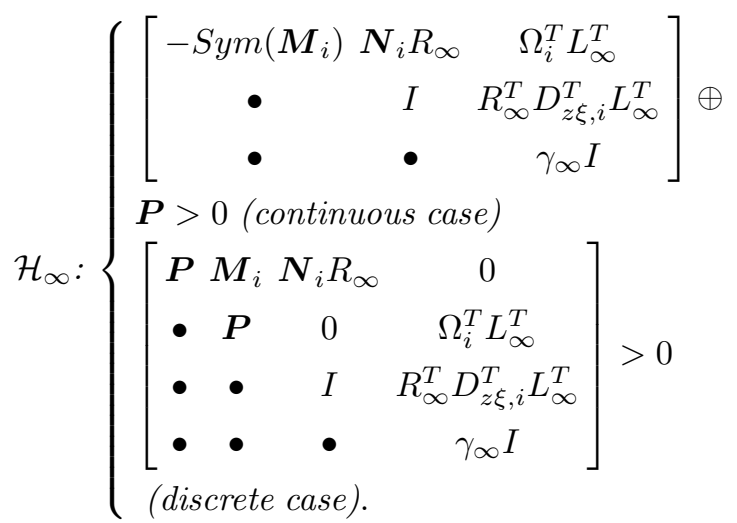

where the matrices $\boldsymbol{M}_{i}, \boldsymbol{N}_{i}, \boldsymbol{P}$, and $\Omega_{i}$ are defined as

$$
\begin{aligned}
& \boldsymbol{M}_{i} \triangleq\left[\begin{array}{c}
\boldsymbol{X}\left(A_{i}+B_{u, i} F\right) \\
\boldsymbol{Y}\left(A_{i}+B_{u, i} F\right)-\boldsymbol{Z}-\boldsymbol{G}\left(C_{y, i}+D_{y u, i} F\right) \\
-\boldsymbol{X} B_{u, i} F \\
\boldsymbol{Z}+\boldsymbol{G} D_{y u, i} F-\boldsymbol{Y} B_{u, i} F
\end{array}\right] \\
& \boldsymbol{N}_{i} \triangleq\left[\begin{array}{c}
\boldsymbol{X} B_{\xi, i} \\
\boldsymbol{Y} B_{\xi, i}-\boldsymbol{G} D_{y \xi, i}
\end{array}\right], \boldsymbol{P} \triangleq\left[\begin{array}{rr}
\boldsymbol{X} \\
\\
\boldsymbol{Y}
\end{array}\right] \\
& \Omega_{i} \triangleq\left[\begin{array}{l}
\left.C_{z, i}+D_{z u, i} F-D_{z u, i} F\right]
\end{array}\right.
\end{aligned}
$$

Furthermore, the controller (15) with $\boldsymbol{A}_{\boldsymbol{c}}=\boldsymbol{Y}^{-1} \boldsymbol{Z}$ and $\boldsymbol{B}_{\boldsymbol{c}}=\boldsymbol{Y}^{-1} \boldsymbol{G}$ achieves (35).

PROOF. For the sake of brevity, only an outline of the 
proof is given. Application of Lemmas 3 and 4 to the closed-loop system matrices results in the bilinear terms $\boldsymbol{P} \overline{\boldsymbol{A}}_{c l}^{\Delta}$, and $\boldsymbol{P} \overline{\boldsymbol{B}}_{c l}^{\Delta}$ from the matrices $\mathcal{M}_{C T}\left(\overline{\boldsymbol{A}}_{c l}^{\Delta}, \overline{\boldsymbol{B}}_{c l}^{\Delta}, \boldsymbol{P}\right)$ and $\mathcal{M}_{D T}\left(\overline{\boldsymbol{A}}_{c l}^{\Delta}, \overline{\boldsymbol{B}}_{c l}^{\Delta}, \boldsymbol{P}\right)$, defined in (9). Clearly, with $\boldsymbol{P}$ defined as in (33) we can write

$$
\begin{aligned}
& \boldsymbol{P} \bar{A}_{c l}^{\Delta}=\left[\begin{array}{c}
\boldsymbol{X}\left(A^{\Delta}+B_{u}^{\Delta} F\right) \\
\boldsymbol{Y}\left(A^{\Delta}+B_{u}^{\Delta} F\right)-\boldsymbol{Y} \boldsymbol{B}_{\boldsymbol{c}}\left(C_{y}^{\Delta}+D_{y u}^{\Delta} F\right)-\boldsymbol{Y} \boldsymbol{A}_{\boldsymbol{c}}
\end{array}\right. \\
& \left.\begin{array}{c}
-\boldsymbol{X} B_{u}^{\Delta} F \\
\boldsymbol{Y} \boldsymbol{A}_{\boldsymbol{c}}+\boldsymbol{Y} \boldsymbol{B}_{\boldsymbol{c}} D_{y u}^{\Delta} F-\boldsymbol{Y} B_{u}^{\Delta} F
\end{array}\right] \\
& \boldsymbol{P} \bar{B}_{c l}^{\Delta}=\left[\begin{array}{c}
\boldsymbol{X} B_{\xi}^{\Delta} \\
\boldsymbol{Y} B_{\xi}^{\Delta}-\boldsymbol{Y} \boldsymbol{B}_{c} D_{y \xi}^{\Delta}
\end{array}\right] .
\end{aligned}
$$

Making the one-to-one change of variables

$$
\left[\begin{array}{llll}
\boldsymbol{Y} & A_{c} & \boldsymbol{Y} & \boldsymbol{B}_{\boldsymbol{c}}
\end{array}\right]=\left[\begin{array}{ll}
Z & G
\end{array}\right]
$$

results in $\boldsymbol{P} \overline{\boldsymbol{A}}_{c l, i}=\boldsymbol{M}_{i}$, and $\boldsymbol{P} \overline{\boldsymbol{B}}_{c l, i}=\boldsymbol{N}_{i}$, with the matrices $\boldsymbol{M}_{i}$ and $\boldsymbol{N}_{i}$ defined as in (39), being linear in the new variables.

\section{Summary of the Approach}

We next summarize the proposed approach to robust dynamic output-feedback controller design.

\section{Algorithm 1 (Robust Output-Feedback Design)}

Use the result in Theorem 7 to find an initially feasible controller, represented by the variables $\left(\boldsymbol{x}_{0}, \boldsymbol{y}_{0}, \gamma_{0}\right)$ related to the corresponding BMI problem (21). Set $\left(\boldsymbol{x}^{*}, \boldsymbol{y}^{*}, \gamma_{U B}^{(0)}\right)=\left(\boldsymbol{x}_{0}, \boldsymbol{y}_{0}, \gamma_{0}\right)$. Solve the relaxed LMI problem (27) to obtain $\gamma_{L B}^{(0)}$. Select the desired precision (relative tolerance) TOL and the maximum number of iterations allowed $k_{\max }$. Set $k=1$.

Step 1. TAKE $\gamma_{k}=\frac{\gamma_{U B}^{(k-1)}+\gamma_{L B}^{(k-1)}}{2}$, AND SOLVE THE PROBLEM $\left(\boldsymbol{x}_{k}, \boldsymbol{y}_{k}\right)=\underset{2}{\arg \min } v_{\gamma_{k}}(\boldsymbol{x}, \boldsymbol{y})$ STARTING WiTH INITIAL CONDITION $\left(\boldsymbol{x}^{*}, \boldsymbol{y}^{*}\right)$.

Step 2. IF $v_{\gamma_{k}}\left(\boldsymbol{x}_{k}, \boldsymbol{y}_{k}\right)=0$ THEN SET $\left(\boldsymbol{x}^{*}, \boldsymbol{y}^{*}, \gamma_{U B}^{(k)}\right)=$ $\left(\boldsymbol{x}_{k}, \boldsymbol{y}_{k}, \gamma_{k}\right)$ ELSE SET $\gamma_{L B}^{(k)}=\gamma_{k}$.

Step 3. IF $\left|\gamma_{U B}^{(k)}-\gamma_{L B}^{(k)}\right|<\operatorname{TOL}\left|\gamma_{U B}^{(k)}\right|$ OR $k \geq k_{\max }$ Then Stop $\left(\left(\boldsymbol{x}^{*}, \boldsymbol{y}^{*}, \gamma_{U B}^{(k)}\right)\right.$ IS THE BEST (LOCALLY) FEASIBLE SOLUTION WITH THE DESIRED TOLERANCE) Else Set $k \leftarrow k+1$ AND GO TO Step 1 .

Note, that $\gamma_{L B}$ at each iteration represents an infeasible value for $\gamma$, while $\gamma_{U B}$ - a feasible one. At each iteration of the algorithm the distance between these two bounds is reduced in two. It should again be noted that if for a given $\gamma_{k}$ the optimal value for the cost function

\begin{tabular}{|l|l|l|}
\hline Parameter: & Sym. & Value: \\
\hline \hline gearbox ratio & $N$ & -260.6 \\
\hline joint angle of inertial axis & $\Omega$ & variable \\
\hline effective joint input torque & $T_{j}^{\text {eff }}$ & variable \\
\hline motor torque constant & $K_{t}$ & 0.6 \\
\hline the damping coefficient & $\beta$ & {$[0.36,0.44]$} \\
\hline deformation torque of gearbox & $T_{\text {def }}$ & variable \\
\hline inertia of the input axis & $I_{m}$ & 0.0011 \\
\hline inertia of the output system & $I_{\text {son }}$ & 400 \\
\hline joint angle of the output axis & $\epsilon$ & variable \\
\hline motor current & $i_{c}$ & variable \\
\hline spring constant & $c$ & {$[117000,143000]$} \\
\hline
\end{tabular}

Table 1

The nominal values of the parameters in the linear model of one joint of the SRM.

$v_{\gamma_{k}}\left(\boldsymbol{x}_{k}, \boldsymbol{y}_{k}\right)$ is nonzero then the algorithm assumes $\gamma_{k}$ as infeasible. Since the algorithm converges to a local minimum it may happen that the original BMI problem is actually feasible for this $\gamma_{k}$ (e.g. corresponding to the global optimum) but the local optimization is unable to confirm feasibility - an effect that cannot be circumvented

\section{An Illustrative Example}

\subsection{Locally Optimal Robust Multiobjective Controller Design}

The example considered consists of a linear model of one joint of a real-life space robot manipulator (SRM) system, taken from (Kanev and Verhaegen, 2000). The state-space model of the system is given by

$$
\begin{aligned}
& \dot{x}(t)=\left[\begin{array}{llcc}
0 & 1 & 0 & 0 \\
0 & 0 & \frac{c}{N^{2} I_{m}} & 0 \\
0 & 0 & 0 & 1 \\
0 & -\frac{\beta}{I_{\text {son }}}-\frac{c}{N^{2} I_{m}}-\frac{c}{I_{\text {son }}}-\frac{\beta}{I_{\text {son }}}
\end{array}\right] x(t)+\left[\begin{array}{c}
0 \\
\frac{K_{t}}{N I_{m}} \\
0 \\
-\frac{K_{t}}{N I_{m}}
\end{array}\right] u(t) \\
& y(t)=\left[\begin{array}{llll}
0 & N & 0 & 0 \\
1 & 0 & 1 & 0
\end{array}\right] x(t)+\left[\begin{array}{l}
1 \\
0
\end{array}\right] \xi(t) \\
& z(t)=\left[\begin{array}{llll}
1 & 0 & 1 & 0
\end{array}\right] x(t)+\xi(t)
\end{aligned}
$$

The system parameters are given in Table 1 . Note that the damping coefficient $\beta$ and the spring constant $c$ are considered uncertain. A Bode plot of the open-loop system for different values of the two uncertain parameters is given in Figure 1.

The objective (see Figure 2) is to find a controller that achieves for all possible values of the uncertain parameters a disturbance rejection of at least 1:100 for constant 

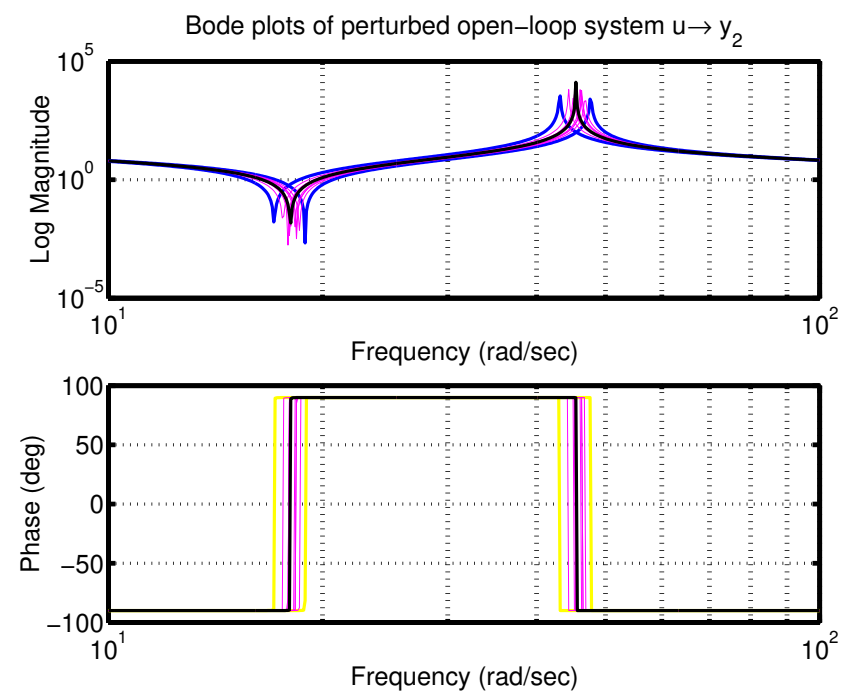

Fig. 1. Bode plot of the perturbed open-loop transfer from $u$ to $y_{2}$.

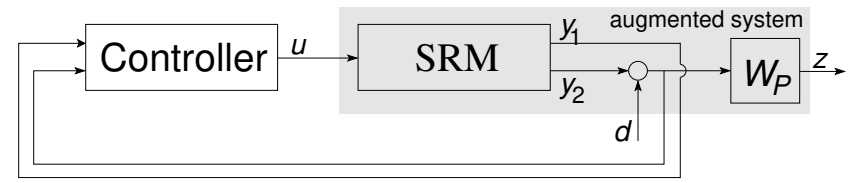

Fig. 2. Closed-loop system with the selected weighting function $W_{p}(s)$.

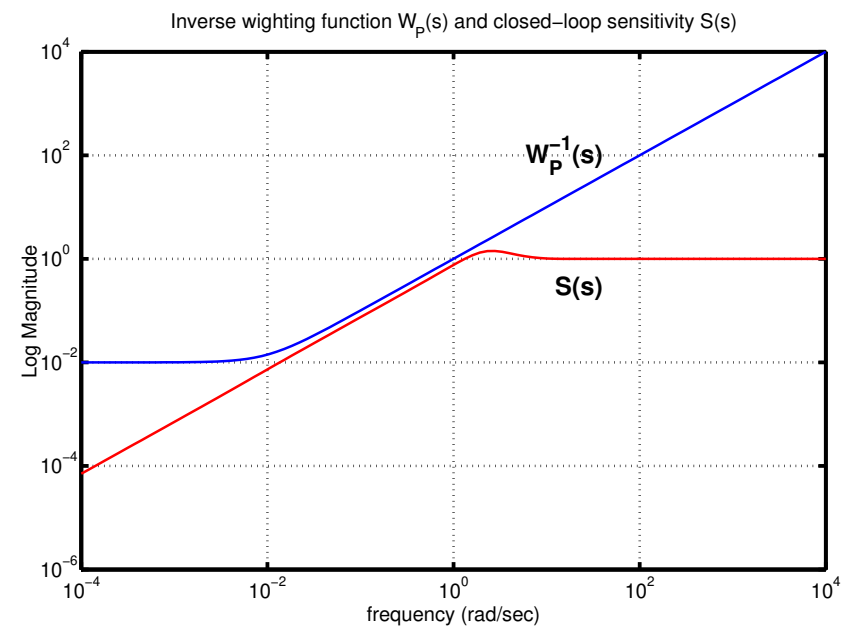

Fig. 3. Sensitivity function of the closed-loop system for the nominal values of the parameters and the inverse of the weighting function $W_{p}$.

disturbances on the shaft angular position (i.e. $z(t)$ ) of the motor (such as, e.g., load), and a bandwidth of at least $1[\mathrm{rad} / \mathrm{sec}]$. This can be achieved by selecting the following performance weighting function (see the upper curve on Figure 3) $W_{p}(s)=1 /(s+0.01)$ and then requiring that $\left\|W_{p}(s) S(s)\right\|_{\infty}<1$ holds for all uncertainties, where $S(s)$ is the transfer function from the disturbance $d$ to the controlled output $z=y_{2}$. In other words, the design specifications would be achieved with a given controller $K(s)$ if the closed-loop transfer function from the disturbance $d$ to the $z$ lies below the Bode magnitude plot of $W_{p}^{-1}(s)$.

It should be noted here that this problem is of a rather large scale: the BMI optimization problem (21) consists of 4 bilinear matrix inequalities, each of dimension $12 \times 12$, and each a function of 95 variables (40 for the controller parameters, and 55 for the closed-loop Lyapunov matrix). Also note, that the number of complicating variables, defined in (Tuan and Apkarian, 2000) as $\min \{\operatorname{dim}(\boldsymbol{x}), \operatorname{dim}(\boldsymbol{y})\}$, in this example equals 40 . This makes it clear that the problem is far beyond the capabilities of the global approaches to solving the underlying BMI problem, which can at present deal with no more than just a few complicating variables.

First, using the result in Theorem 7 an initial controller was found achieving an upper bound of $\gamma_{\infty, \text { init }}=1.0866$, which was subsequently used to initialize the newly proposed BMI optimization (see Algorithm 1). The tolerance of ToL $=10^{-3}$ was selected. The new algorithm converged in 10 iterations to $\gamma_{\infty, N E W}=0.6356$. The computation took about 100 minutes on a computer with a Pentium IV CPU $1500 \mathrm{MHz}$ and 1 Gb RAM.

Next, four other algorithms were tested on this example with the same initial controller, the same tolerance and the same stopping conditions. These algorithms were Rank Minimization Approach (RMA) (Ibaraki and Tomizuka, 2001), the Method of Centers (MC) (Goh et al., 1995), the Path-Following Method (PATH) (Hassibi et al., 1999), and the Alternating coordinate method (DK) (Iwasaki, 1999). The results are summarized in Table 2 . From among these four approaches only two were able to improve the initial controller, namely the MC which achieved $\gamma_{\infty, M C}=0.8114$ in about 610 minutes, and the DK iteration that terminated in about 20 minutes with $\gamma_{\infty, D K}=0.8296$. The MC method was unable to improve the the performance further due to numerical problems. Similar problems were reported in (Fukuda and Kojima, 2001). The PATH converged to an infeasible solution due to the fact that the initial condition is not "close enough" to the optimal one, so that the first order approximation that is made at each iteration is not accurate. Finally, the RMA method was also unable to find a feasible solution.

This experiment shows that after initializing all BMI approaches with the same controller, the newly proposed method outperforms the other compared methods by achieving the lowest value for the cost function. On the other hand, the initial controller itself also achieves a value for the cost function that is rather close to the optimal costs obtained by the DK and the MC methods, i.e. these methods were not able to significantly improve the initial solution. This implies that the initial controller design method could provide a good initial point 


\begin{tabular}{||c|c||}
\hline \hline method & achieved $\gamma_{\text {opt }}$ \\
\hline NEW & 0.6356 \\
\hline RMA & - \\
\hline MC & 0.8114 \\
\hline PATH & infeas. \\
\hline DK & 0.8296 \\
\hline \hline
\end{tabular}

Table 2

Performance achieved by the five local BMI approaches applied to the model of SRM.

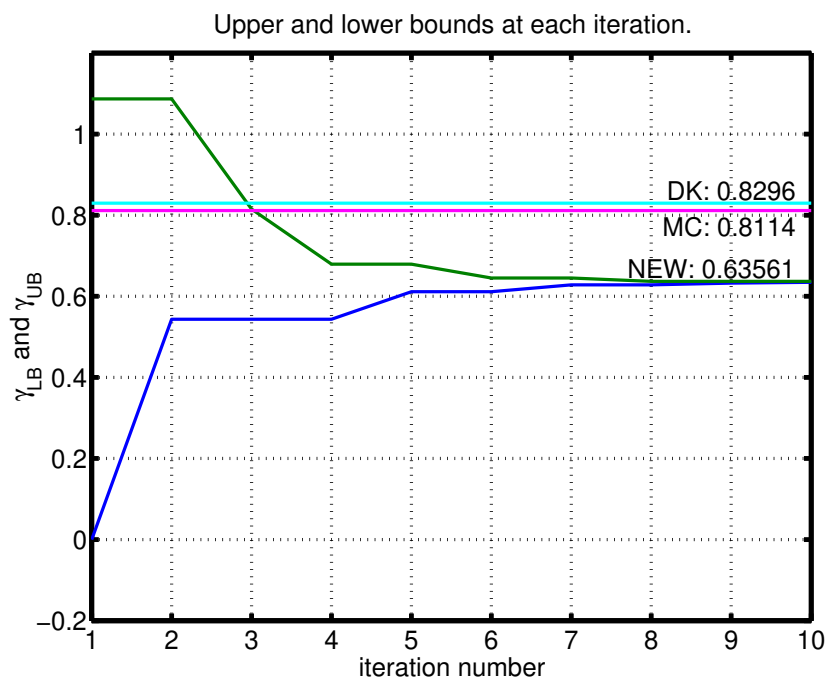

Fig. 4. Upper and lower bounds on $\gamma$ during the BMI optimization.

for starting a local optimization.

For the newly proposed method, the upper and the lower bounds on $\gamma$ at each iteration are plotted in Figure 4. Note that at each iteration the upper bound represents a feasible value for $\gamma$, and the lower bound - an infeasible one. Also plotted on the same figure are the values achieved by the DK iteration and the MC methods. We note that the difficulties that some of the other local approaches experienced is mainly due to the large scale of the problem that causes numerical difficulties and very slow convergence.

The optimal controller obtained after the execution of the newly proposed method has the form (15). With this optimal controller, the closed-loop sensitivity function is depicted in Figure 3, together with the inverse of the selected performance weighting function $W_{p}^{-1}(s)$. It can be seen from the figure that the sensitivity function remains below $W_{p}^{-1}(s)$, implying that the desired robust performance has been achieved.

\section{Conclusions}

In this paper a new approach to the design of locally optimal robust dynamic output-feedback controllers for systems with structured uncertainties was presented. The uncertainty is allowed to have a very general structure and is only assumed to be such that the state-space matrices of the system belong to a certain convex set. The approach is based on BMI optimization that is guaranteed to converge to a locally optimal solution provided that an initially feasible controller is given. This algorithm enjoys the useful properties of computational efficiency and guaranteed convergence to a local optimum. An algorithm for fast computation of an initially feasible controller is also provided and is based on a two-step procedure, where at each step an LMI optimization problem is solved - one to find the optimal state-feedback gain and one to find the remaining state-space matrices of the output-feedback controller. The design objectives considered are $\mathcal{H}_{2}, \mathcal{H}_{\infty}$, and pole-placement in LMI regions. The approach was tested on a model of one joint of a real-life space robotic manipulator, for which a robust $\mathcal{H}_{\infty}$ controller was designed. In addition, the proposed approach was compared to several existing approaches on a simpler BMI optimization and it became clear that it can act as a good alternative for some applications.

\section{Acknowledgements}

This work is sponsored by the Dutch Technology Foundation (STW) under project number DEL.4506.

\section{References}

Apkarian, P., Gahinet, P., 1995. A convex characterization of gain-scheduled $\mathcal{H}_{\infty}$ controllers. IEEE Transactions on Automatic Control 40(5), 853-864.

Balas, G., Doyle, J., Glover, K., Packard, A., Smith, R., 1998. $\mu$-Analysis and Synthesis Toolbox - For Use with Matlab. MUSYN Inc. and MathWorks Inc.

Beran, E., Vandenberghe, L., Boyd, S., 1997. A global BMI algorithm based on the generalized benders decomposition. In: Proceedings of the European Control Conference. Brussels, Belgium.

Boyd, S., Ghaoui, L. E., Feron, E., Balakrishnan, V., 1994. Linear Matrix Inequalities in System and Control Theory. SIAM Studies in Applied Mathematics, volume 15, Philadelphia, PA.

Calafiore, G., Polyak, B., 2001. Stochastic algorithms for exact and approximate feasibility of robust LMIs. IEEE Transactions on Automatic Control 46(11), 1755-1759.

Chilali, M., Gahinet, P., Apkarian, P., 1999. Robust pole placement in LMI regions. IEEE Transactions on Automatic Control 44(12), 2257-2269.

Collins, E. G., Sadhukhan, D., Watson, L. T., 1999. Robust controller synthesis via non-linear matrix in- 
equalities. International Journal of Control 72(11), 971-980.

Cuzzola, F. A., Ferrante, A., 2001. Explicit formulas for LMI-based $\mathcal{H}_{2}$ filtering and deconvolution. Automatica 37(9), 1443-1449.

Doyle, J. C., 1983. Synthesis of robust controllers and filters. In: Proceedings of the IEEE Conference on Decision and Control. San Antonio, TX.

Forsgren, A., 2000. Optimality conditions for nonconvex semidefinite programming. Mathematical Programming 88(1), 105-128.

Fukuda, M., Kojima, M., 2001. Branch-and-cut algorithms for the bilinear matrix inequality eigenvalue problem. Computational Optimization and Applications 19, 79-105.

Gahinet, P., 1996. Explicit controller formulas for LMIbased $\mathcal{H}_{\infty}$ synthesis. Automatica 32(7), 1007-1014.

Gahinet, P., Nemirovski, A., Laub, A., Chilali, M., 1995. LMI Control Toolbox User's Guide. MathWorks, Inc.

Geromel, J. C., 1999. Optimal linear filtering under parameter uncertainty. IEEE Transactions on Signal Processing 47(1), 168-175.

Geromel, J. C., Bernussou, J., de Oliveira, M. C., 1999. $\mathcal{H}_{2}$-norm optimization with constrained dynamic output feedback controllers: Decentralized and reliable control. IEEE Transactions on Automatic Control 44(7), 1449-1454.

Geromel, J. C., Bernussou, J., Garcia, G., de Oliveira, M. C., 2000. $\mathcal{H}_{2}$ and $\mathcal{H}_{\infty}$ robust filtering for discretetime linear systems. SIAM Journal on Control and Optimization 38(5), 1353-1368.

Geromel, J. C., de Oliveira, M. C., 2001. $\mathcal{H}_{2}$ and $\mathcal{H}_{\infty}$ robust filtering for convex bounded uncertain systems. IEEE Transactions on Automatic Control 46(1), 100107.

Goh, K.-C., Safonov, M. G., Ly, J. H., 1996. Robust synthesis via bilinear matrix inequalities. International Journal of Robust and Nonlinear Control 6(9-10), 1079-1095.

Goh, K.-C., Safonov, M. G., Papavassilopoulos, G. P., 1995. Global optimization for the biaffine matrix inequality problem. Journal of Global Optimization 7(4), 365-380.

Grigoradis, K. M., Skelton, R. E., 1996. Low-order control design for LMI problems using alternating projection methods. Automatica 32(8), 1117-1125.

Hassibi, A., How, J., Boyd, S., 1999. A path-following method for solving BMI problems in control. In: Proceedings of the American Control Conference. San Diego, CA, pp. 1385-1389.

Hol, C. W. J., Scherer, C. W., van der Meché, E. G., Bosgra, O. H., 2003. A nonlinear SPD approach to fixed order controller synthesis and comparison with two other methods applied to an active suspension system. European Journal of Control 9(1), 11-26.

Ibaraki, S., Tomizuka, M., 2001. Rank minimization approach for solving BMI problems with random search. In: Proceedings of the American Control Conference. Arlington, VA, pp. 1870-1875.
Iwasaki, T., 1999. The dual iteration for fixed order control. IEEE Transations on Automatic Control 44(4), $783-788$.

Iwasaki, T., Rotea, M., 1997. Fixed order scaled $\mathcal{H}_{\infty}$ synthesis. Optimal Control Applications \& Methods 18(6), 381-398.

Iwasaki, T., Skelton, R. E., 1995. The XY-centering algorithm for the dual LMI problem: A new approach to fixed order control design. International Journal of Control 62(6), 1257-1272.

Kanev, S., Verhaegen, M., 2000. Controller reconfiguration for non-linear systems. Control Engineering Practice 8(11), 1223-1235.

Kose, I. E., Jabbari, F., 1999a. Control of LPV systems with partly measured parameters. IEEE Transactions on Automatic Control 44(3), 658-663.

Kose, I. E., Jabbari, F., 1999b. Robust control of linear systems with real parametric uncertainty. Automatica 35(4), 679-687.

Kothare, M. V., Balakrishnan, V., Morari, M., 1996. Robust constrained model predictive control using linear matrix inequalities. Automatica 32(10), 1361-1379.

Leibfritz, F., 2001. An LMI-based algorithm for designing suboptimal static $\mathcal{H}_{2} / \mathcal{H}_{\infty}$ output feedback controllers. SIAM Journal on Control \& Optimization 39(6), 1711-1735.

Leibfritz, F., Mostafa, E. M. E., 2002. An intereior point constraint trust region method for a special class of nonlinear semidefinite programming problems. SIAM Journal on Optimization 12(4), 1047-1074.

Li, D. H., Fukushima, M., 2001. On the global convergence of the BFGS method for nonconvex unconstrained optimization problems. SIAM Journal on Optimization 11(4), 1054-1064.

Mahmoud, M. S., Xie, L. H., 2000. Positive real analysis and synthesis of uncertain discrete-time systems. IEEE Transactions on Circuits and Systems-Part I: Fundamental Theory and Applications 47(3), 403406.

Masubuchi, I., Ohara, A., Suda, N., 1998. LMI-based controller synthesis: A unified formulation and solution. International Journal of Robust and Nonlinear Control 8(8), 669-686.

Oliveira, M. C., Bernussou, J., Geromel, J. C., 1999. A new discrete-time robust stability condition. Systems \& Control Letters 37(4), 261-265.

Oliveira, M. C., Geromel, J. C., Bernussou, J., 2002. Extended $\mathcal{H}_{2}$ and $\mathcal{H}_{\infty}$ norm characterizations and controller parametrizations for discrete-time systems. International Journal of Control 75(9), 666-679.

Palhares, R. M., de Souza, C. E., Peres, P. L. D., 2001. Robust $\mathcal{H}_{\infty}$ filter design for uncertain discretetime state-delayed systems: An LMI approach. IEEE Transactions on Signal Processing 49(8), 1696-1703.

Palhares, R. M., Ramos, D. C. W., Peres, P. L. D., 1996. Alternative LMIs characterization of $\mathcal{H}_{2}$ and central $\mathcal{H}_{\infty}$ discrete-time controllers. In: Proc. of the 35th IEEE Conference on Decision and Control. Kobe, Japan, pp. 1459-1496. 
Palhares, R. M., Takahashi, R. H. C., Peres, P. L. D., 1997. $\mathcal{H}_{2}$ and $\mathcal{H}_{\infty}$ guaranteed costs computation for uncertain linear systems. International Journal of Systems Science 28(2), 183-188.

Peres, P. L. D., Palhares, R. M., 1995. Optimal $\mathcal{H}_{\infty}$ state feedback control for discrete-time linar systems. In: Second Latin American Seminar on Advanced Control, LASAC'95. Chile, pp. 73-78.

Scherer, C., Gahinet, P., Ghilali, M., 1997. Multiobjective output-feedback control via LMI optimization. IEEE Transactions on Automatic Control 42(7), 896911.

Toker, O., Özbay, H., 1995. On the NP-hardness of solving bilinear matrix inequalities and simultaneous stabilization with static output feedback. In: Proceedings of the American Control Conference. Seattle, WA, pp. 2525-2526.

Tuan, H. D., Apkarian, P., 2000. Low nonconvexity-rank bilinear matrix inequalities: Algorithms and applications in robust controller and structure designs. IEEE Transations on Automatic Control 45(11), 2111-2117.

Tuan, H. D., Apkarian, P., Hosoe, S., Tuy, H., 2000a. D.C. optimization approach to robust control: Feasibility problems. International Journal of Control 73(2), 89-104.

Tuan, H. D., Apkarian, P., Nakashima, Y., 2000b. A new Lagrangian dual global optimization algorithm for solving bilinear matrix inequalities. International Journal of Robust and Nonlinear Control 10, 561-578.

VanAntwerp, J. G., Braatz, R. D., 2000. A tutorial on linear and bilinear matrix inequalities. Journal of Process Control 10, 363-385.

VanAntwerp, J. G., Braatz, R. D., Sahinidis, N. V., 1997. Globally optimal robust control for systems with time-varying nonlinear perturbations. Computers and Chemical Engineering 21, S125-S130.

Xie, L., Fu, M., de Souza, C. E., 1992. $\mathcal{H}_{\infty}$ control and quadratic stabilization of systems with parameter uncertainty via output feedback. IEEE Transactions on Automatic Control 37(8), 1253-1256.

Yamada, Y., Hara, S., 1998. Global optimization for the $\mathcal{H}_{\infty}$ control with constant diagonal scaling. IEEE Transactions on Automatic Control 43(2), 191-203.

Yamada, Y., Hara, S., Fujioka, H., 2001. $\epsilon$-feasibility for $\mathcal{H}_{\infty}$ control problems with constant diagonal scalings. Transactions of the Society of Instrument and Control Engineers E-1(1), 1-8.

Zhou, K., Doyle, J., 1998. Essentials of Robust Control. Prentice-Hall.

Zhou, K., Khargonekar, P. P., Stoustrup, J., Niemann, H. H., 1995. Robust performance of systems with structured uncertainties in state-space. Automatica 31(2), 249-255. 